\title{
In-situ Studies of High Temperature Thermal Batteries: A Perspective
}

\author{
Julia L. Payne ${ }^{1 *}$, Kyriakos Giagloglou ${ }^{1}$, George M. Carins ${ }^{1}$, Christina J. Crouch ${ }^{1,2}$, \\ Julia D. Percival ${ }^{2}$, Ronald I. Smith ${ }^{3}$, Richard K. B. Gover ${ }^{2}$ and John T. S. Irvine ${ }^{1}$ \\ ${ }^{1}$ School of Chemistry, Faculty of Science, University of St Andrews, St Andrews, United Kingdom, ${ }^{2}$ AWE \\ Aldermaston, Reading, United Kingdom, ${ }^{3}$ ISIS Facility STFC Rutherford Appleton Laboratory, Didcot, United Kingdom
}

Here we present a perspective on in-situ studies of high temperature batteries. We focus on a primary battery technology- the thermal battery- which possesses a molten salt electrolyte. We discuss aspects of sample environment design, data collection and will briefly look at some case studies. We aim to highlight the importance of using in-situ techniques in studying electrochemical devices such as high temperature batteries.

Keywords: in-situ, neutron diffraction, thermal battery, transition metal sulfides, high temperature batteries

\section{OPEN ACCESS}

Edited by:

Neeraj Sharma

University of New South Wales,

Australia

Reviewed by:

Xiangyu Zhao,

Nanjing Tech University, China Verónica Palomares,

Universidad del País Vasco, Spain Donna Claire Arnold,

University of Kent, United Kingdom

${ }^{*}$ Correspondence:

Julia L. Payne

jlp8@st-andrews.ac.uk

Specialty section:

This article was submitted to

Energy Storage,

a section of the journal

Frontiers in Energy Research

Received: 04 May 2018 Accepted: 25 October 2018 Published: 20 November 2018

Citation:

Payne JL, Giagloglou K, Carins GM, Crouch CJ, Percival JD, Smith RI, Gover RKB and Irvine JTS (2018) In-situ Studies of High Temperature

Thermal Batteries: A Perspective. Front. Energy Res. 6:121. doi: 10.3389/fenrg.2018.00121

\section{INTRODUCTION}

In recent years, the study of battery technologies using in-situ techniques has become increasingly important (Sharma et al., 2015). This has been driven by a desire for the precise understanding of the processes occurring during battery operation, which may be used to optimize batteries or inform the future synthetic targets of new battery materials with improved performance. This has been accompanied by improvements in the design of cells which allow collection of both electrochemical data and the data of interest (Bergstrom et al., 1998; Berg and Thomas, 1999; Sharma and Peterson, 2012; Roberts et al., 2013; Biendicho et al., 2014; Sharma et al., 2015; Brant et al., 2016). Techniques such as powder X-ray diffraction (PXRD), powder neutron diffraction (PND), Nuclear Magnetic Resonance (NMR) and X-ray absorption spectroscopy (XAS) have all been used (Bergstrom et al., 1998; Berg and Thomas, 1999; Balasubramanian et al., 2001; Morcrette et al., 2003; Yoon et al., 2003; Borkiewicz et al., 2012; Herklotz et al., 2013; Roberts et al., 2013; Biendicho et al., 2014; Brant et al., 2016; Kim et al., 2016; Gu et al., 2017; Pecher et al., 2017). This has been accompanied by improvements in instrumentation at synchrotron and neutron facilities, along with a willingness to accommodate unusual sample environments and set-ups on the beamline.

With the lithium-ion battery revolutionizing the portable electronics market in recent years, it is unsurprising that the majority of these in-situ studies have focused on batteries which operate at room temperature. However, batteries are required for a wide range of applications and there remain several battery technologies that have not been studied using in-situ techniques.

One battery technology which we are currently investigating is the thermal battery. Thermal batteries are primary batteries which are used in emergency power supplies in aircraft, ejector seats, and other military applications (Guidotti and Masset, 2006). Applications which utilize thermal batteries require a constant power to be drawn over a length of time, so as a result, materials that operate via conversion reactions, rather than intercalation reactions are usually preferred. Typically they consist of a cathode, anode, molten salt electrolyte, separator and a pyrotechnic ignition source to heat the battery (Masset and Guidotti, 2007, 2008a,b; Guidotti and Masset, 2008). Due to the use of a molten salt electrolyte, thermal batteries must operate at a temperature above the melting point of the electrolyte, which means that they often operate at temperatures 
close to $500^{\circ} \mathrm{C}$. The high temperature operation, in addition to the fact that air/moisture-sensitive transition metal sulfides are the most commonly used cathodes, means that structural studies of this battery technology would be best performed using in-situ techniques, and would require a complex cell design. To the best of our knowledge no such studies have been reported.

Our current research interests have focused on the in-situ study of thermal batteries which use transition metal sulfides as the cathode material, $\mathrm{Li}_{13} \mathrm{Si}_{4}$ as the anode material, $\mathrm{KCl}-\mathrm{LiCl}$ eutectic as an electrolyte and $\mathrm{MgO}$ as the separator. In particular the transition metal disulfides, $\mathrm{MS}_{2}$ (where $\mathrm{M}=\mathrm{Fe}, \mathrm{Co}$, and $\mathrm{Ni}$ ) are the most commonly used cathode materials in thermal batteries and they offer a particularly intriguing study, as the discharge mechanisms are thought to be different depending on the transition metal present in the cathode, with only cells using $\mathrm{FeS}_{2}$ as a cathode forming lithiated compounds upon battery discharge (Preto et al., 1983).

\section{EXPERIMENTAL CONSIDERATIONS}

\section{Design of Sample Environment}

The purpose of our experiments was to carry out combined battery discharge and powder neutron diffraction studies. A sample environment for combined conductivity and neutron diffraction studies of hydride ion conductors was designed by one of us, so this sample environment was modified to enable it to be used for in-situ thermal battery studies instead (Payne et al., 2017; Carins et al., in preparation). A photograph of the sample environment is shown in Figure 1A. This sample environment is able to maintain an inert atmosphere around the sample, which is important when looking at air/moisture sensitive species. During each experiment, Argon gas was continuously flowed through the sample environment.

The sample environment consists of a closed-end quartz tube, which has thinned walls near the sample area to allow for beam entry and exit. There is a gas inlet/outlet on the top of the tube. Wires for electrical connections run from the top of the tube to the sample holder and are sheathed in $\mathrm{Al}_{2} \mathrm{O}_{3}$.

By modifying the sample environment using vanadium plates as both current collectors and sample holder (Figure 1B) we were able to simplify the data analysis by reducing the number of phases that will be observed in the diffraction pattern [vanadium's very low neutron scattering length $(-0.39 \mathrm{fm})$ compared to those of the atomic species making up the other phases present means that it contributes very little to the neutron diffraction pattern of the battery]. Neutron diffraction is particularly appropriate when studying lithium-containing materials in the presence of much higher Z-number atoms because unlike X-ray scattering (which depends on the number of electrons) neutrons scatter from the atomic nucleus and this results in the scattering power of lithium being comparable to the other species in the battery. Recesses in the vanadium plates hold the sample in position for optimum beam convergence on the sample. A diagram of the vanadium plate sample holder is shown in Figure 1B.

The design of an equivalent cell for the equivalent $\mathrm{X}$-ray diffraction experiment would be non-trivial. A suitable current collector is difficult to find and existing sample environments
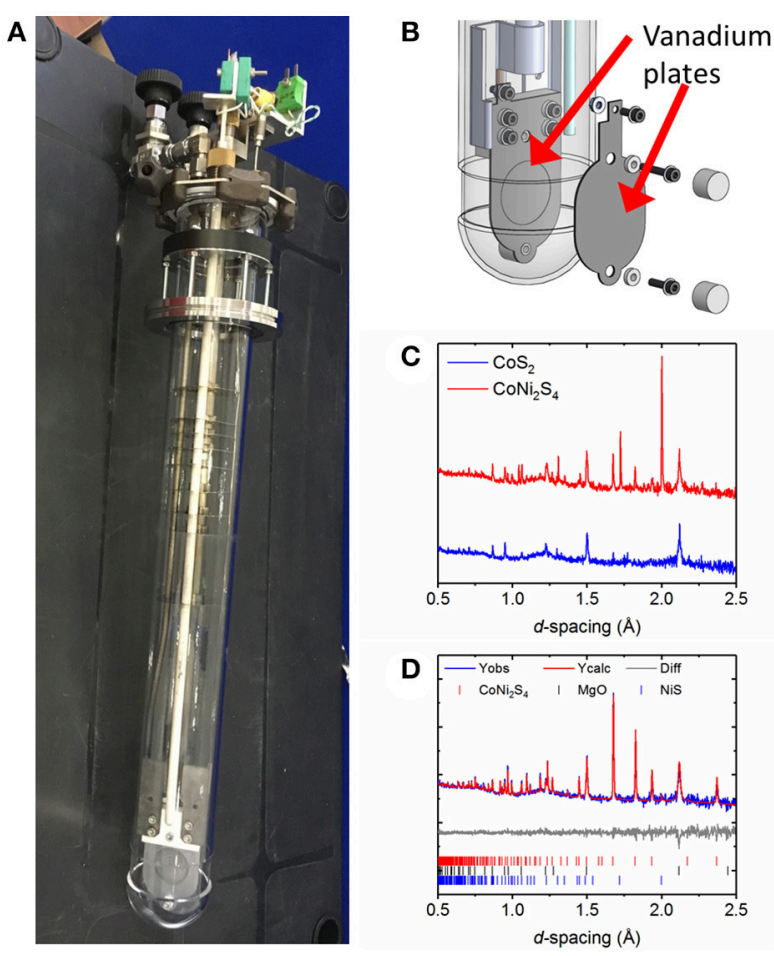

FIGURE 1 | (A) Photograph of the in-situ sample environment used for studies of thermal batteries which allows the flow of an inert gas during the course of the experiment to maintain an inert atmosphere. (B) Shows a diagram of vanadium plate current collector which doubles as a sample holder. Screws are used to help hold the sample in place. (C) Comparison of powder diffraction data obtained in $5 \mathrm{~min}$ on the Polaris diffractometer for two cells using two different cathode materials: $\mathrm{CoS}_{2}$ and $\mathrm{CoNi}_{2} \mathrm{~S}_{4}$ (D) multi-phase Rietveld fit to data collected in 5 min on the Polaris diffractometer during the discharge of a thermal battery which uses $\mathrm{CoNi}_{2} \mathrm{~S}_{4}$ as the cathode material. Please note that differences in intensity of the $\mathrm{CoNi}_{2} \mathrm{~S}_{4}$ data are due to the data being collected at different states of discharge. Broad features in the background of the diffraction pattern are due to the amorphous quartz outer container of the in-situ sample environment and molten salt eutectic.

designed to work at room temperature use beryllium windows which would oxidize at the high temperatures required for this study.

\section{Choice of Instrument}

Our in-situ experiments have been carried out on the Polaris diffractometer at the ISIS facility, Rutherford Appleton Laboratory, UK. Polaris is a high flux, medium resolution diffractometer, which is particularly suited to fast data collection and unusual sample environments, making it the ideal choice for the in-situ experiment. The recent upgrade of Polaris enables the collection of good quality diffraction data, even with very small samples (Lindsay-Scott et al., 2014).

\section{Data Collection Protocols}

With any in-situ experiment, there is balance to be achieved between the electrochemistry and the structural data that will be collected. Neutron powder diffraction requires larger samples than the corresponding X-ray diffraction experiment. In our first 
experiments we decided to preserve the electrochemistry as much as possible, which resulted in the use of a thinner pellet shaped cell ( $\sim 1-2 \mathrm{~mm}$ thickness) than would normally be required for neutron diffraction. This led to limitations in the parameters that could be extracted from multi-phase Rietveld refinement, such as meaningful thermal parameters. Figure $\mathbf{1 C}$ shows the data quality from $5 \mathrm{~min}$ neutron powder diffraction data collections on $\mathrm{CoS}_{2}$ and $\mathrm{CoNi}_{2} \mathrm{~S}_{4}$ based cells. Much better data quality could be obtained for the $\mathrm{CoNi}_{2} \mathrm{~S}_{4}$ cell which benefitted from both being a slightly thicker sample, along with the higher neutron scattering length of nickel $(10.3 \mathrm{fm})$ in comparison to cobalt $(2.49 \mathrm{fm})$ and sulfur $(2.85 \mathrm{fm})$. Data were analyzed by carrying out multi-phase Rietveld refinements. Figure 1D shows a multiphase Rietveld fit to a 5 min data set collected during discharge of a thermal battery which used $\mathrm{CoNi}_{2} \mathrm{~S}_{4}$ as the cathode material. This indicates that good quality data can often be obtained in times as short as a few minutes when using neutron diffraction, but it is dependent on the sample being studied.

During an in-situ experiment, it is important to minimize the contributions to the diffraction pattern from parts of the sample environment, and we were able to do this by adjusting the dimensions of the incident neutron beam to match the geometry of the thermal battery sample as well as utilizing neutron shielding materials (boron nitride) to cover parts of the sample environment that may scatter (and would contribute to the diffraction pattern). The reliability of a spallation neutron source can also be challenging during such in-situ experiments, where short interruptions to the beam due to minor accelerator faults may result in "gaps" in the data, but fortunately for our experiments carried out to date, this has not been problematic.

In terms of carrying out the battery discharge, two approaches have been used. Firstly, potentiostatic discharge has been used, where data are collected at fixed isopotentials. The isopotentials are determined by an ex-situ battery discharge in our home laboratory before our in-situ experiment, in order to ensure that diffraction data are collected at the key plateaux on the discharge profile. Longer diffraction datasets are collected at the isopotentials, and on switching between isopotentials faster data collection takes place. Alternatively, galvanostatic discharge maybe carried out, during which a large number of diffraction patterns are collected continuously over short periods of time (e.g., 5 mins each), and which subsequently may be summed to obtain higher quality datasets on plateaux in the discharge profile. We have had success with both types of measurement, but we found that it was difficult to get meaningful capacities from our in-situ experiments to date.

\section{Sample Preparation}

The samples for the experiments were prepared by sequentially pressing the powders for the anode, electrolyte/separator and cathode into pellets. The sequential pressing offers the opportunity to make good contacts between each layer. It is then relatively straightforward to handle the pellet and load it into the in-situ sample environment inside the glovebox. The eutectic of choice for the electrolyte is the $\mathrm{KCl}-\mathrm{LiCl}$ eutectic (melting point $=352^{\circ} \mathrm{C}$ ). At room temperature, peaks from the electrolyte are present in the neutron powder diffraction pattern, but on heating above the melting point of the electrolyte, the peaks disappear, which again simplifies the data analysis by reducing the number of phases observed in the neutron diffraction pattern.

\section{CASE STUDIES: THERMAL BATTERIES USING TRANSITION METAL SULFIDES, AS CATHODES}

As discussed earlier, transition metal disulfides $\mathrm{MS}_{2}$ (where $\mathrm{M}=$ $\mathrm{Fe}, \mathrm{Co}$, and $\mathrm{Ni}$ ) are some of the most commonly used cathode materials for thermal batteries, and indeed it was these materials which have formed the basis of our first in-situ studies on thermal batteries (Payne et al., 2017; Payne et al., in preparation). In addition, our recent synthetic and electrochemical work has looked at some other transition metal sulfides as potential cathodes for thermal batteries (Giagloglou et al., 2016, 2017; Giagloglou et al., in preparation).

Our first in-situ study looked at the discharge of a thermal battery which used $\mathrm{NiS}_{2}$ (a conversion material) as the cathode, $\mathrm{Li}_{13} \mathrm{Si}_{4}$ as the anode, $\mathrm{KCl}-\mathrm{LiCl}$ eutectic as the electrolyte and $\mathrm{MgO}$ as the separator (Payne et al., 2017). This experiment was carried out at $520^{\circ} \mathrm{C}$, which to the best of our knowledge, is the highest temperature in-situ battery experiment carried out to date. Figure 2 shows some of the results from this experiment, including a film plot of the diffraction data collected during battery discharge, a plot of the evolution of phase fraction and discharge profile over the course of the experiment and finally the changes in crystal structure of Ni-containing species during discharge. In our study, we were able to correlate the evolution of crystalline phases with plateaux observed in the battery discharge profile and a total of four nickel containing phases were observed in the diffraction patterns. A film plot of the diffraction data shows evolution of the crystalline phases upon discharge and is shown in Figure 2A. Rietveld refinement was used to extract phase fractions from the diffraction data. This allows us to correlate the phase fractions with the electrochemical data, and in particular with the plateau in the voltage discharge profile. Figure 2B shows the evolution of phase fraction and cell voltage during discharge. This shows that the plateau in the voltage discharge profile at $\sim 1.7 \mathrm{~V}$ was due to the $\mathrm{NiS}_{2}$ to NiS transformation, whilst the plateau at $\sim 1.4-1.3 \mathrm{~V}$ was due to the NiS to $\mathrm{Ni}_{3} \mathrm{~S}_{2}$ transformation. We did not observe $\mathrm{Ni}_{7} \mathrm{~S}_{6}$, which had previously been observed in cells examined at room temperature and this led us to propose a new discharge mechanism for $\mathrm{NiS}_{2}$ when it is used as a cathode (Payne et al., 2017). The structures of the Ni-containing phases that were identified during discharge are shown in Figure 2C. We should also point out that we also observed changes in cell parameter of the NiS phase, which provides evidence for a region of solid solution, and although the changes in composition of this phase could not be accurately determined it is possible that this change in cell parameter was due to either a non-stoichiometric $\mathrm{Ni}_{1-x} \mathrm{~S}$ phase or some Li incorporation in NiS. It was particularly useful to collect synchrotron powder X-ray diffraction data on the cathode after our in-situ neutron diffraction data, as this enabled conclusive identification of all cell components present, 


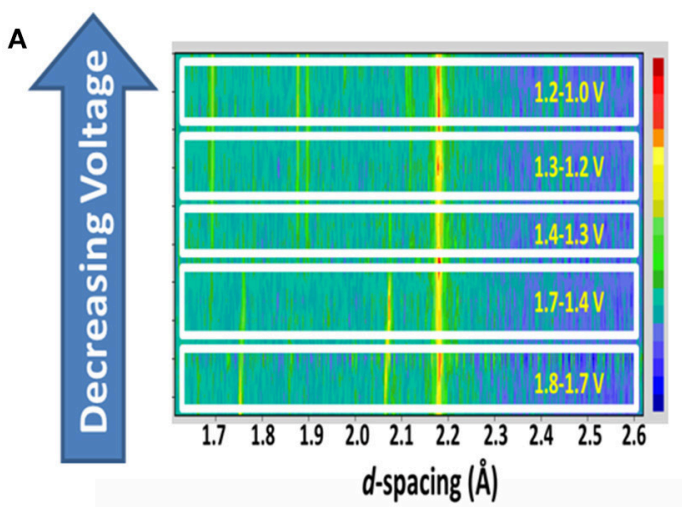

B

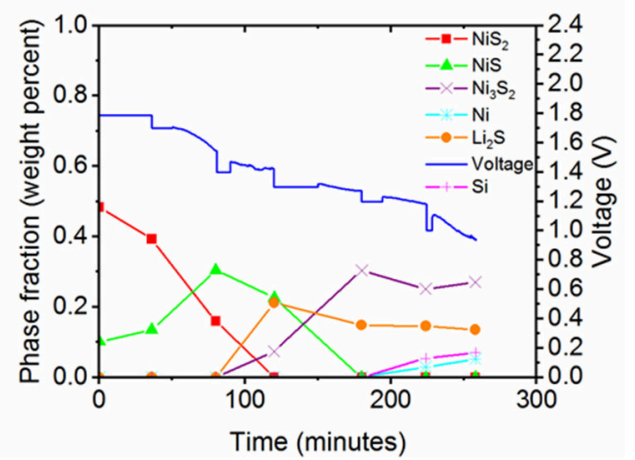

C

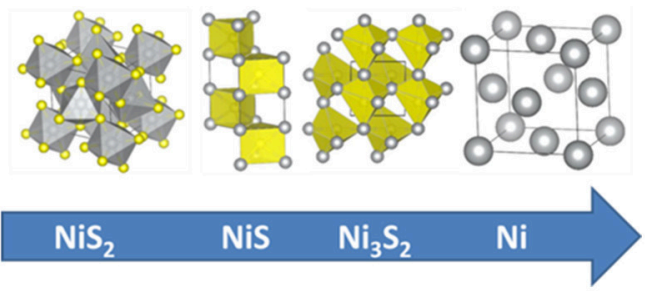

FIGURE 2 | Results from the in-situ thermal battery discharge using $\mathrm{NiS}_{2}$ as the cathode (Payne et al., 2017). (A) Film plot of neutron diffraction data collected during battery discharge. Please note that the $y$-axis is not a linear function of time. (B) Evolution of phase fraction and voltage over the course of the experiment. (C) Crystal structures of $\mathrm{Ni}$ containing species observed during the course of battery discharge. (A,B) Copyright Wiley- $\mathrm{VCH}$ Verlag $\mathrm{GmbH}$ \& Co. KGaA and are reproduced from Payne et al. (2017) with permission.

as we benefitted from the high intensity and resolution of the synchrotron source. This emphasizes the importance of using complementary techniques (where possible) to enhance the understanding of the system under study.

$\mathrm{Li}_{13} \mathrm{Si}_{4}$ is an anode commonly used in thermal batteries. However, in our in-situ studies to date, the combination of a large unit cell and low symmetry of the $\mathrm{Li}_{13} \mathrm{Si}_{4}$ structure, which is then pressed into a pellet, means that it is particularly difficult to study the changes in the structure of the anode material during discharge. Although the lithium silicides have been well-studied by a variety of techniques, including single crystal diffraction and NMR, much less is known about their high temperature behavior, including the high temperature crystallography (Key et al., 2009, 2011; Zeilinger and Faessler, 2013; Zeilinger et al., 2013a,b). It is also possible that $\mathrm{Li}_{13} \mathrm{Si}_{4}$ (and related phases) are largely amorphous during battery discharge, as electrochemically driven amorphisation is known to occur in lithium ion batteries upon lithium uptake when $\mathrm{Si}$ is used as an anode (Limthongkul et al., 2003a,b). The challenges in studying the anode material highlights that often questions still remain after an in-situ experiment, meaning that such experiments may often need follow up studies, in order to fully understand the system of interest.

Restricting in-situ studies to diffraction based techniques means that it can be easy to neglect any components which are amorphous. In the case of the thermal battery, the presence of molten salt electrolytes can simplify data analysis, due to the reduction in the number of crystalline phases observed in the diffraction pattern at device operating temperature. However, there may be occasions when we are interested in the amount of amorphous material in the battery. The use of a crystalline ceramic separator in thermal batteries is particularly useful as it can be used as an internal standard to quantify the amorphous content the battery. The importance of gaining an understanding of non-crystalline phases has recently been highlighted in a study of the in-situ synthesis of the $\mathrm{Li}_{7} \mathrm{La}_{3} \mathrm{Zr}_{2} \mathrm{O}_{12} \mathrm{Li}$-ion conductor. This quantified the fraction of molten/amorphous phases present in this system and found that the presence of a carbonate melt assists the synthesis of $\mathrm{Li}_{7} \mathrm{La}_{3} \mathrm{Zr}_{2} \mathrm{O}_{12}$ (Rao et al., 2015).

\section{OTHER HIGH-TEMPERATURE BATTERY TECHNOLOGIES: SODIUM METAL HALIDE BATTERIES}

We should point out that thermal batteries are not the only high temperature battery technology. Sodium-metal halide batteries, which are also often referred to as "ZEBRA" (ZeroEmission Battery Research Activities) cells are another high temperature battery technology which have been studied using in-situ techniques (Zinth et al., 2015, 2016). These cells typically operate at around $300^{\circ} \mathrm{C}$ and have also been studied by in-situ neutron diffraction (Zinth et al., 2015). Spatially resolved neutron diffraction data were collected at different points within the cathode of such a cell, enabling reaction fronts to be monitored as they move through the battery (Zinth et al., 2015). In-situ neutron tomography and radiography have been used to probe the changes in morphology of the cell components during battery cycling (Zinth et al., 2016). The phase evolution in sodium metal halide batteries and the presence of intermediates have also been studied using in-situ energy dispersive X-ray diffraction and this has allowed both spatial and kinetic information to be obtained on the battery charge/discharge process (Russenbeek et al., 2011).

\section{CONCLUSIONS}

Although most in-situ studies of batteries have focused on batteries which operate at room temperature, other battery technologies, such as the thermal battery and the sodium metal halide battery, operate at higher temperatures and require insitu studies to be carried out at elevated temperature, in order to gain a better understanding of the processes taking place in them. Our recent in-situ work has successfully probed and 
shed light on the processes occurring in thermal batteries during discharge at their high operating temperatures of around $500^{\circ} \mathrm{C}$. To the best of our knowledge we believe that these are the highest temperature in-situ battery studies carried out to date. We should also point out that studies using complementary techniques such as synchrotron PXRD post battery testing offer advantages such as high resolution and intensity, which can help to unambiguously identify products formed during battery discharge. Although improvements can be envisioned and there are still many questions to be answered, this work paves the way for future high temperature in-situ studies of batteries.

\section{AUTHOR CONTRIBUTIONS}

JLP co-wrote the article, carried out experiments, and analyzed data. KG prepared samples and carried out experiments. GC

\section{REFERENCES}

Balasubramanian, M., Sun, X., Yang, X. Q., and McBreen, J. (2001). In situ Xray diffraction and $\mathrm{X}$-ray absorption studies of high-rate lithium-ion batteries. Power Sources J. 92, 1-8. doi: 10.1016/s0378-7753(00)00493-6

Berg, H., and Thomas, J. O. (1999). Neutron diffraction study of electrochemically delithiated $\mathrm{LiMn}_{2} \mathrm{O}_{4}$ spinel. Solid State Ionics, 126, 227-234.doi: 10.1016/s0167-2738(99)00235-0

Bergstrom, O., Andersson, A. M., Edstrom, K., and Gustafsson, T. (1998). A neutron diffraction cell for studying lithium-insertion processes in electrode materials. J. Appl. Crystallogr. 31, 823-825. doi: 10.1107/s00218898980 $0538 \mathrm{x}$

Biendicho, J. J., Roberts, M., Offer, C., Noreus, D., Widenkvist, E., Smith, R. I., et al. (2014). New in-situ neutron diffraction cell for electrode materials. Power Sources J. 248, 900-904. doi: 10.1016/j.jpowsour.2013.09.141

Borkiewicz, O. J., Shyam, B., Wiaderek, K. M., Kurtz, C., Chupas, P. J., and Chapman, K. W. (2012). The AMPIX electrochemical cell: a versatile apparatus for in situ X-ray scattering and spectroscopic measurements. J. Appl. Crystallogr. 45, 1261-1269. doi: 10.1107/s0021889812042720

Brant, W. R., Roberts, M., Gustafsson, T., Biendicho, J. J., Hull, S., Ehrenberg, H., et al. (2016). A large format in operando wound cell for analysing the structural dynamics of lithium insertion materials. Power Sources J. 336, 279-285. doi: 10.1016/j.jpowsour.2016.10.071

Giagloglou, K., Payne, J. L., Crouch, C., Gover, R. K. B., Connor, P. A., and Irvine, J. T. S. (2016). Zirconium trisulfide as a promising cathode material for Li primary thermal batteries. J. Electrochem. Soc. 163, A3126-A3130. doi: 10.1149/2.1351614jes

Giagloglou, K., Payne, J. L., Crouch, C., Gover, R. K. B., Connor, P. A., and Irvine, J. T. S. (2017). Synthesis and electrochemical study of $\mathrm{CoNi}_{2} \mathrm{~S}_{4}$ as a novel cathode material in a primary Li thermal battery. J. Electrochem. Soc. 164, A2159-A2163. doi: 10.1149/2.1171709jes

Gu, Q. F., Kimpton, J. A., Brand, H. E. A., Wang, Z. Y., and Chou, S. L. (2017). Solving key challenges in battery research using in situ synchrotron and neutron techniques. Adv. Energy Mater. 7:1602831. doi: 10.1002/aenm.201602898

Guidotti, R. A., and Masset, P. (2006). Thermally activated ("thermal") battery technology - Part I: An overview. Power Sources J. 161, 1443-1449. doi: 10.1016/j.jpowsour.2006.06.013

Guidotti, R. A., and Masset, P. J. (2008). Thermally activated ("thermal") battery technology - Part IV. Anode materials. Power Sources J. 183, 388-398. doi: 10.1016/j.jpowsour.2008.04.090

Herklotz, M., Scheiba, F., Hinterstein, M., Nikolowski, K., Knapp, M., Dippel, A. C., et al. (2013). Advances in in situ powder diffraction of battery materials: a case study of the new beamline P02.1 at DESY, Hamburg. J. Appl. Crystallogr. 46, 1117-1127. doi: 10.1107/s0021889813013551

Key, B., Bhattacharyya, R., Morcrette, M., Seznéc, V., Tarascon, J. M., and Grey, C. P. (2009). Real-time NMR investigations of structural changes in developed the sample environment and carried out experiments. CC carried out experiments. JDP and RS carried out experiments and co-wrote manuscript. RG and JI co-wrote manuscript and oversaw research.

\section{FUNDING}

We thank AWE and EPSRC (EP/P007821/1) for supporting this programme of research.

\section{ACKNOWLEDGMENTS}

We thank the STFC for provision of neutron diffraction beamtime and Diamond Light Source for the provision of X-ray diffraction beam-time. silicon electrodes for lithium-ion batteries. J. Am. Chem. Soc. 131, 9239-9249. doi: $10.1021 /$ ja 8086278

Key, B., Morcrette, M., Tarascon, J. M., and Grey, C. P. (2011). Pair Distribution function analysis and solid state NMR studies of silicon electrodes for lithium ion batteries: understanding the (De)lithiation mechanisms. J. Am. Chem. Soc. 133, 503-512. doi: 10.1021/ja108085d

Kim, T., Song, B. H., Lunt, A. J. G., Cibin, G., Dent, A. J., Lu, L., et al. (2016), Operando X-ray absorption spectroscopy study of atomic phase reversibility with wavelet transform in the lithium-rich manganese based oxide cathode. Chem. Mater. 28, 4191-4203. doi: 10.1021/acs.chemmater.6b00522

Limthongkul, P., Jang, Y. I., Dudney, N. J., and Chiang, Y. M. (2003a). Electrochemically-driven solid-state amorphization in lithium-metal anodes. Power Sources J. 119, 604-609. doi: 10.1016/s0378-7753(03)00303-3

Limthongkul, P., Jang, Y. I., Dudney, N. J., and Chiang, Y. M. (2003b). Electrochemically-driven solid-state amorphization in lithium-silicon alloys and implications for lithium storage. Acta Mater. 51, 1103-1113. doi: 10.1016/s1359-6454(02)00514-1

Lindsay-Scott, A., Dobson, D., Nestola, F., Alvaro, M., Casati, N., Liebske, C., et al. (2014). Time-of-flight neutron powder diffraction with milligram samples: the crystal structures of $\mathrm{NaCoF}_{3}$ and $\mathrm{NaNiF}_{3}$ post-perovskites. J. Appl. Crystallogr. 47, 1939-1947. doi: 10.1107/s1600576714021803

Masset, P., and Guidotti, R. A. (2007). Thermal activated (thermal) battery technology - Part II. Molten salt electrolytes. Power Sources J. 164, 397-414. doi: $10.1016 /$ j.jpowsour.2006.10.080

Masset, P. J., and Guidotti, R. A. (2008a). Thermal activated ("thermal") battery technology - Part IIIa: $\mathrm{FeS}_{2}$ cathode material. Power Sources J. 177, 595-609. doi: 10.1016/j.jpowsour.2007.11.017

Masset, P. J., and Guidotti, R. A. (2008b). Thermal activated ("thermal") battery technology. Part IIIb. Sulfur and oxide-based cathode materials. Power Sources J. 178, 456-466. doi: 10.1016/j.jpowsour.2007.11.073

Morcrette, M., Leriche, J. B., Patoux, S., Wurm, C., and Masquelier, C. (2003). In situ X-ray diffraction during lithium extraction from rhombohedral and monoclinic $\mathrm{Li}_{3} \mathrm{~V} 2(\mathrm{PO} 4) 3$. ECS Solid State Lett. 6, A80-A84. doi: $10.1149 / 1.1563871$

Payne, J. L., Percival, J. D., Giagloglou, K., Crouch, C. J., Carins, G. M., Smith, R. I., et al. (2017). In-situ thermal battery discharge using $\mathrm{NiS}_{2}$ as a cathode material. Chemelectrochem. 4, 1916-1923. doi: 10.1002/celc.201700095

Pecher, O., Carretero-Gonzalez, J., Griffith, K. J., and Grey, C. P. (2017). Materials' methods: NMR in battery research. Chem. Mater. 29, 213-242. doi: 10.1021/acs.chemmater.6b03183

Preto, S. K., Tomczuk, Z., Vonwinbush, S., and Roche, M. F. (1983). Reactions of $\mathrm{FeS}_{2}, \mathrm{CoS}_{2}$, and $\mathrm{NiS}_{2}$ electrodes in molten LiCl-KCl electrolytes. J. Electrochem. Soc. 130, 264-273. doi: 10.1149/1.2119692

Rao, R. P., Gu, W. Y., Sharma, N., Peterson, V. K., Avdeev, M., and Adams, S. (2015). In situ neutron diffraction monitoring of $\mathrm{Li}_{7} \mathrm{La}_{3} \mathrm{Zr}_{2} \mathrm{O}_{12}$ formation: toward a rational synthesis of garnet solid 
electrolytes. Chem. Mater. 27, 2903-2910. doi: 10.1021/acs.chemmater. 5 b00149

Roberts, M., Biendicho, J. J., Hull, S., Beran, P., Gustafsson, T., Svensson, G., et al. (2013). Design of a new lithium ion battery test cell for insitu neutron diffraction measurements. Power Sources J. 226, 249-255. doi: 10.1016/j.jpowsour.2012.10.085

Russenbeek, J., Gao, Y., Zhong, Z., Croft, M., Jisrawi, N., Ignatov, A., et al. (2011). In situ X-ray diffraction of prototype sodium metal halide cells Time and space electrochemical profiling. Power Sources J. 196, 2332-2339. doi: 10.1016/j.jpowsour.2010.10.023

Sharma, N., Pang, W. K., Guo, Z. P., and Peterson, V. K. (2015). In situ powder diffraction studies of electrode materials in rechargeable batteries. Chemsuschem, 8, 2826-2853. doi: 10.1002/cssc.2015 00152

Sharma, N., and Peterson, V. K. (2012). In situ neutron powder diffraction studies of lithium-ion batteries. J. Solid State Electrochem. 16, 1849-1856. doi: 10.1007/s10008-011-1567-5

Yoon, W. S., Grey, C. P., Balasubramanian, M., Yang, X. Q., and McBreen, J. (2003). In situ X-ray absorption spectroscopic study on $\mathrm{LiNi}_{0.5} \mathrm{Mn}_{0.5} \mathrm{O}_{2}$ cathode material during electrochemical cycling. Chem. Mater. 15, 3161-3169. doi: $10.1021 / \mathrm{cm} 030220 \mathrm{~m}$

Zeilinger, M., Benson, D., Haussermann, U., and Fassler, T. F. (2013a). Single crystal growth and thermodynamic stability of $\mathrm{Li}_{17} \mathrm{Si}_{4}$. Chem. Mater. 25, 1960-1967. doi: 10.1021/cm40 $0612 \mathrm{k}$
Zeilinger, M., and Fässler, T. F. (2013). Revision of the $\mathrm{Li}_{13} \mathrm{Si}_{4}$ structure. Acta Cryst. E. 69, i81-i82. doi: 10.1107/S1600536813029759

Zeilinger, M., Kurylyshyn, I. M., Haussermann, U., and Fassler, T. F. (2013b). Revision of the Li-Si phase diagram: discovery and single-crystal X-ray structure determination of the high-temperature phase $\mathrm{Li}_{4.11} \mathrm{Si}$. Chem. Mater. 25, 4623-4632. doi: 10.1021/cm4029885

Zinth, V., Schulz, M., Seidlmayer, S., Zanon, N., Gilles, R., and Hofmann, M. (2016). Neutron tomography and radiography on a sodium metal halide cell under operating conditions. J. Electrochem. Soc. 163, A838-A845. doi: 10.1149/2.0181606jes

Zinth, V., Seidlmayer, S., Zanon, N., Crugnola, G., Schulz, M., Gilles, R., et al. (2015). In situ spatially resolved neutron diffraction of a sodium metal halide battery. J. Electrochem. Soc. 162, A384-A391. doi: 10.1149/2.0421503jes

Conflict of Interest Statement: The authors declare that the research was conducted in the absence of any commercial or financial relationships that could be construed as a potential conflict of interest.

Crown Copyright (C) 2018 Authors: Payne, Giagloglou, Carins, Crouch, Percival, Smith, Gover and Irvine. This is an open-access article distributed under the terms of the Creative Commons Attribution License (CC BY). The use, distribution or reproduction in other forums is permitted, provided the original author(s) and the copyright owner(s) are credited and that the original publication in this journal is cited, in accordance with accepted academic practice. No use, distribution or reproduction is permitted which does not comply with these terms. 